\title{
Diffuse Scattering and Defect Structure Simulations
}

\author{
A cook book using \\ the program DISCUS
}

\author{
Reinhard B. Neder \\ Department of Physics, University of Erlangen-Nürnberg \\ Thomas Proffen \\ Los Alamos National Laboratory
}




\section{Contents}

1 Introduction 1

2 How to run DISCUS 3

3 Making computer crystals $\quad 7$

3.1 Storing the structure $\ldots \ldots \ldots \ldots \ldots \ldots$

3.2 Small assemblies of atoms $\ldots \ldots \ldots \ldots \ldots \ldots$

3.3 Generating the unit cell . . . . . . . . . . . . . . . 14

3.4 Generating extended crystal structures . . . . . . . 19

3.5 Unit cell transformations . . . . . . . . . . . . . . 21

3.6 General symmetry operations . . . . . . . . . . . . 24

3.7 Creating molecules . . . . . . . . . . . . . . . 27

3.8 Example: Distorted perovskite $\ldots \ldots \ldots \ldots \ldots . \ldots 28$

3.9 Bibliography . . . . . . . . . . . . . 32

4 Simulating experimental data 35

4.1 Single-crystal scattering $\ldots \ldots \ldots \ldots \ldots$. . . . . . 35

4.1 .1 Finite size effects . . . . . . . . . . . . 36

4.1 .2 Coherence . . . . . . . . . . . . . . . 38

4.2 Powder diffraction . . . . . . . . . . . . . . 39

4.2.1 Complete integration . . . . . . . . . . 40

4.2 .2 Debye formula . . . . . . . . . . . . . 41

4.3 Atomic pair distribution function $\ldots \ldots \ldots \ldots \ldots 43$

4.3.1 Calculating the PDF from a model . . . . . . . 43

4.3.2 Modeling of thermal motion . . . . . . . . 45

4.4 Properties of the Fourier transform . . . . . . . . . . 48

4.5 Bibliography . . . . . . . . . . . . . . . . 49

5 Correlations and creating short-range order 53

5.1 What are correlations? . . . . . . . . . . . . . 53

5.2 Monte Carlo simulations . . . . . . . . . . . . . 54

5.3 Creating chemical short-range order . . . . . . . . . 55

5.4 Creating displacement disorder $\ldots \ldots \ldots \ldots \ldots 56$

5.4 .1 Simple spring . . . . . . . . . . . . . 56

5.4 .2 Lennard-Jones potential . . . . . . . . . . 56

5.4 .3 Bond angles . . . . . . . . . . . . . . 57

5.5 Example: Chemical short-range order . . . . . . . . 57

vii 
5.6 Example: Distortions . . . . . . . . . . . . . . . 62

5.7 Bibliography ..................... 65

6 Creating modulations $\quad 69$

6.1 Density waves . . . . . . . . . . . . . . 70

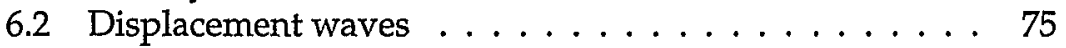

6.3 Finite waves . . . . . . . . . . . . . . . 79

6.4 Pitfalls when simulating modulations . . . . . . . 84

6.5 Bibliography . . . . . . . . . . . . . 85

7 Creating structures with stacking faults 87

7.1 Types of stacking faults . . . . . . . . . . . . 87

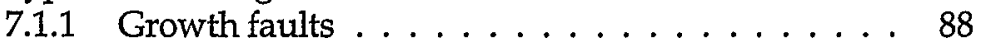

7.1 .2 Deformation faults ............. 89

7.1.3 Stacking fault parameters . . . . . . . . . 89

7.2 Notations for stacking sequences . . . . . . . . . . . . 90

7.3 Reciprocal space of layered structures . . . . . . . . . . . 91

7.4 Algorithms to simulate stacking faults . . . . . . . . . 93

7.4.1 Growth faults . . . . . . . . . . . . . . . 94

7.4 .2 Deformation faults . . . . . . . . . . . 95

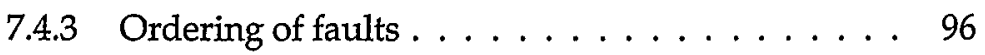

7.5 Example: Growth faults . . . . . . . . . . . . . 97

7.6 Example: Deformation faults . . . . . . . . . . . 101

7.7 Example: Wurtzite and zincblende structures . . . . . . 103

7.8 Example: Short-range ordered faults . . . . . . . . 104

7.9 Bibliography . . . . . . . . . . . . . . 108

8 Creating domain structures 113

8.1 Introduction . . . . . . . . . . . . . . 113

8.2 Domain types ...................... 114

8.3 Definitions for a domain .............. 115

8.4 Ordering and distribution of domains . . . . . . . . . . . 121

8.5 Domain formation in Perovskites . . . . . . . . . . . . . 124

8.6 Example: Urea inclusion compounds . . . . . . . . . . . 128

8.7 Bibliography . . . . . . . . . . . . 135

9 Creating nanoparticles 139

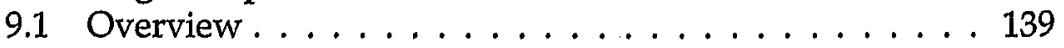

9.2 Creating simple particles . . . . . . . . . . . . . . . 140

9.3 PDF of nanoparticles . . . . . . . . . . . . . . . . 145

9.4 Creating core-shell particles . . . . . . . . . . . . . 150

9.5 Carbon nanotubes . . . . . . . . . . . . . . 156

9.6 Bibliography . . . . . . . . . . . . . . . 159

10 Analyzing disordered structures 161

10.1 Visualizing structures $\ldots \ldots \ldots 16 \ldots$

10.2 Occupancies . . . . . . . . . . . . . 165

10.3 Finding neighbors . . . . . . . . . . . . 166

10.4 Distortions . . . . . . . . . . . . . . . . 167 
10.5 Calculating correlations . . . . . . . . . . . . . 168

10.5.1 Occupational correlations . . . . . . . . . . . 168

10.5.2 Displacement correlations . . . . . . . . . 170

10.5.3 Correlation fields . . . . . . . . . . . . . 171

10.6 Bond valence sums . . . . . . . . . . . . . . 171

10.7 Bibliography . . . . . . . . . . . . . . . . . 172

11 Refining disordered structures 175

11.1 Reverse Monte Carlo method . . . . . . . . . . . . 175

11.2 Length-scale dependent PDF refinements . . . . . . . . 178

11.3 Refining parameters of a disorder model . . . . . . 180

11.3.1 The program Diffev . . . . . . . . . . . . . 183

11.3.2 Required size of the simulated structure . . . . . 186

11.3.3 Example: Simple disordered structure . . . . . . . 188

11.3.4 Example: ZnSe Nanoparticles . . . . . . . . . . 195

11.4 Bibliography . . . . . . . . . . . . . . . . . . . . . 201

A Appendix 205

A.1 Contents of the CD-ROM . . . . . . . . . . . . 205

A.2 Installation . . . . . . . . . . . . . . 205

A.3 Functional list of commands . . . . . . . . . . . 207

A.4 Answers to exercises . . . . . . . . . . . . . . 210

A.5 DISCUS bibliography . . . . . . . . . . . . . 222

$\begin{array}{ll}\text { Index } & 227\end{array}$ 